\title{
Sistema de monitoreo de señales en tierra usando la Estación Terrena Satelital UPTC
}

\section{Signal monitoring system using the UPTC Satellite Ground Station}

\author{
DOI: http://dx.doi.org/10.17981/ingecuc.15.1.2019.04
}

Artículo de Investigación Científica. Fecha de Recepción: 20/07/2018. Fecha de Aceptación: 22/01/2019.

\author{
Jairo Alonso Mesa Lara \\ Universidad Pedagógica y Tecnológica de Colombia. Tunja, (Colombia) \\ jairo.mesa@uptc.edu.co \\ Jenny Amparo Rosales Agredo \\ Universidad Pedagógica y Tecnológica de Colombia. Tunja, (Colombia) \\ jenny.rosales@uptc.edu.co \\ María Paula Maury Atencia \\ Universidad Pedagógica y Tecnológica de Colombia. Tunja, (Colombia) \\ maria.maury@uptc.edu.co
}

J. A. Mesa Lara, J. A. Rosales Agredo y M. P. Maury Atencia, "Sistema de monitoreo de señales en tierra usando la Estación Terrena Satelital UPTC”, INGE CUC, vol. 15, no. 1, pp. 36-44, 2019. DOI: http://doi.org/10.17981/ingecuc.15.1.2019.04

\section{Resumen}

Introducción- La Estación Terrena Satelital (ETS), corresponde a una infraestructura tecnológica conformada por dispositivos de hardware y software que permite la comunicación desde tierra con un satélite.

Objetivo- Este articulo tiene como propósito central describir las fases desarrolladas con la implementación de un sistema de monitoreo remoto de señales en tierra, para el análisis de variables metereológicas, y de esta manera, aumentar el porcentaje de utilización de los dispositivos que conforman la infraestructura de la Estación Terrena Satelital de la Universidad Pedagógica y Tecnológica de Colombia (ETS-UPTC).

Metodología- El diseño metodológico permitió validar el incremento en el porcentaje de uso diario de la ETS-UPTC. Permitió la identificación de las aplicaciones susceptibles a ser monitoreadas en tierra, la descripción de la estructura general del sistema, la adaptación del nodo sensor y el tratamiento de datos para su posterior visualización en el nodo coordinador.

Resultados- Se logró desarrollar un nodo sensor, cuya implementación y adaptación con la ETS-UPTC permitió incrementar su nivel de uso diario en un $20 \%$ aproximadamente.

Conclusiones- La integración del sistema de monitoreo de variables meteorológicas con la ETS-UPTC, sirven como una alternativa, para ser replicada en otras estaciones terrenas satelitales construidas bajo el diseño tradicional monolítico, o como referente para implementar otras soluciones de monitoreo, que exijan el uso de diferentes áreas de conocimiento en su diseño e implementación. El desarrollo del proyecto, se constituye en un aporte social que la Universidad Pedagógica y Tecnológica de Colombia puede ofrecer a los departamentos de Boyacá y Casanare, a través de la consolidación y distribución de la información sobre el comportamiento de variables meteorológicas, datos que podrían ser utilizados para el sistema de alertas tempranas y prevención de riesgos.

Palabras clave- Estación Meteorológica; Estación Terrena Satelital ETS-UPTC; Monitoreo Remoto de Señales en Tierra; Nodo Sensor; Porcentaje de Uso; Variables Meteorológicas.

\begin{abstract}
Introduction- The Satellite Earth Station, corresponds to a technological infrastructure made up of hardware and software devices that allow communication from the ground with a satellite.

Objective- The main purpose of this article is to describe the phases developed with the implementation of a remote monitoring system of ground signals, for the analysis of meteorological variables, and thus increase the percentage of use of the devices that make up the infrastructure of Estación Terrena Satelital de la Universidad Pedagógica y Tecnológica de Colombia (ETS-UPTC).

Methodology - The methodological design allowed to validate the increase in the percentage of daily use of the ETS-UPTC. It allowed the identification of the applications susceptible to be monitored on land, the description of the general structure of the system, the adaptation of the sensor node and the treatment of data for its later visualization in the coordinating node.
\end{abstract}

Results- It was possible to develop a sensor node, whose implementation and adaptation with the ETSUPTC allowed to increase its level of daily use by approximately $20 \%$.

Conclusions- The integration of the monitoring system of meteorological variables with the ETS-UPTC, serve as an alternative, to be replicated in other satellite earth stations built under the traditional monolithic design, or as a reference to implement other monitoring solutions that require the use of different areas of knowledge in their design and implementation. The development of the project constitutes a social contribution that the Universidad Pedagógica y Tecnológica de Colombia can offer to the departments of Boyacá and Casanare, through the consolidation and distribution of information on the behavior of meteorological variables, data that could be used for the early warning system and risk prevention.

Keywords- Weather Station; ETS-UPTC Satellite Earth Station; Remote Monitoring of Signals on Earth; Sensor Node; Percentage of Use; Meteorological Variables. 


\section{INTRODUCCIÓN}

La Estación Terrena Satelital (ETS), comprende una infraestructura tecnológica conformada por dispositivos de hardware y software, que permite la comunicación desde la tierra con un satélite [1].

El presente artículo, describe el sistema creado para el monitoreo de señales en tierra, aumentando de este modo el porcentaje de utilización de los dispositivos que conforman la Estación Terrena Satelital en la Universidad Pedagógica y Tecnológica de Colombia (ETS-UPTC).

Se han realizado trabajos de investigación relacionados con estaciones terrenas enfocados a establecimiento de enlaces satelitales [2]. Países como Argentina [3], cuentan con estaciones terrenas satelitales propias y extranjeras que prestan entre otros servicios soporte a unidades satelitales móviles, gestión del monitoreo y control de satélites, mantenimiento y reparación de equipamiento, operaciones de envío y recepción de señales de diferentes satélites. Por su parte, Brasil posee cuatro estaciones terrenas satelitales que hacen parte del Instituto Nacional de Investigación Espacial de Brasil (INPE), dichas estaciones se usan para el seguimiento, recepción, procesamiento y distribución de datos provenientes de satélites [4]. Otros países sudamericanos, tales como Ecuador, Chile, Venezuela, Perú y Bolivia le han apostado a la implementación de estaciones terrenas [3].

La revisión de la literatura especializada, permite identificar que se han realizado inversiones de tiempo y esfuerzo en constituir infraestructuras de estaciones terrenas, sin embargo, se han limitado a la realización de funciones satelitales. Si bien es cierto, la investigación aeroespacial ha llevado a que algunos países de Sudamérica construyan y pongan en órbita sus propios satélites, pero no todos tienen los recursos para lograrlo y las infraestructuras permanecen subutilizadas; este análisis contextual, refuerza la necesidad de explorar otras posibles funcionalidades como aplicaciones en tierra para este tipo de estaciones. En Estados Unidos, China, Ecuador y México se han diseñado e implementado sistemas de monitoreo de señales, utilizando las bandas UHF y VHF en las áreas de ciencias de la salud, medio ambiente y seguridad.

En Colombia, instituciones de educación superior como la Universidad Sergio Arboleda [5], Universidad Distrital Francisco José de Caldas [6], Universidad Surcolombiana [6], Fundación Universitaria Panamericana [7] y la Universidad Pedagógica y Tecnológica de Colombia [2] han realizado proyectos relacionados con diseño e implementación de estaciones terrenas con funciones exclusivas de seguimiento a satélites. A nivel nacional, diferentes universidades en ciudades como Medellín, Bogotá y Barranquilla, han realizado proyectos relacionados con monitoreo de señales desde una estación o central de monitoreo, sin abarcar el componente satelital.
La Universidad Pedagógica y Tecnológica de Colombia ha realizado diferentes proyectos de investigación relacionados con la Estación Terrena Satelital, cuyos resultados y aspectos más relevantes se encuentran en diferentes trabajos, tales como el diseño del sistema de comunicaciones para la estación terrena [8], montaje e implementación de la estación terrena satelital para el seguimiento a satélites [9], análisis del desempeño del protocolo AX.25 en el enlace de comunicaciones de la estación [2] y en el marco de ese trabajo se propuso un modelo para el sistema de posicionamiento de las antenas [10]. Posteriormente, se realizó un diseño de software para permitir el direccionamiento autónomo de las antenas de la estación empleando técnicas de inteligencia artificial [11].

Tomando como referencia las investigaciones anteriormente enunciadas y los resultados que arrojaron, se implementó la Estación Terrena Satelital ETSUPTC, siguiendo el diseño tradicional monolítico [2] y actualmente se cuenta con antenas para las bandas VHF, UHF y S, transceptores, modem y un mecanismo de posicionamiento formado por servomotores con su respectiva unidad de control. Esta estación realiza funciones de seguimiento (tracking) a satélites, ubicados en la órbita terrestre baja (Low Earth Orbit - LEO) con el uso del software de predicción Orbitron y recepción de señales en la banda de muy alta frecuencia (Very High Frecuency - VHF) mediante un arreglo de antenas tipo Eggbeater crossboom y Antena S-Band, un amplificador de bajo ruido (LowNoise Amplifier - LNA) y un sistema de radio que actúa como transceptor y modem.

Para estimar el tiempo de uso diario en las funciones mencionadas de la ETS se empleó Orbitron, obteniendo una ventana de observación de $6 \mathrm{minu}$ tos aproximadamente para cada satélite. Desde esta estación, se pueden conectar en promedio 8 satélites diarios, lo que genera un tiempo de uso de $50 \mathrm{minu}$ tos, correspondiente a un porcentaje estimado de $4 \%$. Sin embargo, este porcentaje de uso podría incrementarse, dada la inversión hecha en los elementos que conforman la infraestructura de la ETS-UPTC.

El objetivo del proyecto se centró en la posibilidad de optimizar el uso de la infraestructura ETS-UPTC, y en la necesidad de ampliar la gama de aplicaciones que, desde los semilleros y grupos de investigación de la Facultad de ingeniería del alma mater, sean susceptibles a ser desarrollados y sirvan como complemento a la academia.

La investigación permitió la identificación de las aplicaciones para monitoreo de sistemas en tierra, la creación de un circuito denominado nodo sensor, cuya unidad de comunicación permitió el envío de datos de las variables meteorológicas temperatura y humedad relativa, datos que fueron recopilados en el nodo coordinador. Con la integración de los dos nodos se contribuyó al incremento del uso diario de la ETS-UPTC. 
Este artículo, está estructurado en cinco etapas, en las cuales se describen las aplicaciones que pueden ser implementadas para el monitoreo de señales en tierra, el esquema general del sistema de captura de variables meteorológicas, el diseño del nodo sensor, la comunicación entre el nodo sensor y el nodo coordinador, así como el tratamiento de los datos para su posterior visualización en el nodo coordinador. Por último, se presentan los principales resultados, conclusiones y referencias derivadas de la consulta especializada de estudios asociados.

\section{Metodología}

El desarrollo del presente estudio, se basó en el método de investigación inductivo, con un alcance exploratorio y descriptivo; fue enmarcado en esta clasificación teniendo en cuenta los conceptos expuestos por Sampieri, Fernández y Baptista [12]. Todas las etapas fueron abordadas con un enfoque cualitativo y a continuación se describe detalladamente cada una de ellas.

\section{A. Etapa Uno}

En esta etapa de la investigación, se identificaron las aplicaciones susceptibles de ser monitoreadas en tierra utilizando la infraestructura de la estación y se seleccionó como estudio de caso el monitoreo de algunas variables capturadas en la estación meteorológica de la ETS-UPTC.

Inicialmente, se identificaron los rangos de frecuencia de operación de la ETS-UPTC, estos están determinados por las frecuencias legalmente permitidas en Colombia [13] y por las características propias de las antenas, las cuales se muestran en la Tabla 1.

Tabla 1. Rango de FREcuencias Para OPERACIÓN DE LA ESTACIÓN TERRENA UPTC

\begin{tabular}{|c|c|}
\hline Rango de frecuencias & Uso \\
\hline VHF: $145-148 \mathrm{MHz}$ & Aficionados \\
\hline UHF: $430-440 \mathrm{MHz}$ & Aficionados \\
\hline S: $2300-2450 \mathrm{MHz}$ & Aficionados \\
\hline S: $2400-2483,5 \mathrm{MHz}$ & Aplicaciones ICM* \\
\hline * Industriales, Científicas y Médicas \\
\hline
\end{tabular}

Fuente: Autores.

En la Tabla 2, se presenta un resumen de aplicaciones del monitoreo, que se podrían implementar con una infraestructura como la que tiene la ETSUPTC, las cuales han sido clasificadas de acuerdo a cada una de las áreas de conocimiento asociadas. El resumen fue formulado, tomando como referencia la revisión de casos de éxito en las áreas de Ciencias
Agrícolas, Ciencias Médicas y de la Salud, Ciencias Naturales, Ciencias de la Educación, Ingeniería y Tecnología, con bandas de frecuencia compatibles y que podrían ser recibidas, procesadas y posteriormente compartidas desde la estación terrena satelital.

Tabla 2. Resumen de aplicaciones de Monitoreo

\begin{tabular}{|l|l|}
\hline \multicolumn{1}{|c|}{$\begin{array}{c}\text { Área de } \\
\text { Conocimiento }\end{array}$} & \multicolumn{1}{|c|}{ Aplicación } \\
\hline \multirow{3}{*}{$\begin{array}{l}\text { Ciencias } \\
\text { Agrícolas }\end{array}$} & $\begin{array}{l}\text { Monitoreo de signos vitales de animales } \\
{[14]}\end{array}$ \\
\cline { 2 - 2 } & Agricultura de precisión [15] \\
\hline \multirow{2}{*}{$\begin{array}{l}\text { Ciencias de la } \\
\text { Salud }\end{array}$} & Telemetría biomédica [16] [17] [18] [19] \\
\cline { 2 - 2 } $\begin{array}{l}\text { Ciencias } \\
\text { Naturales }\end{array}$ & $\begin{array}{l}\text { Monitoreo médico [16] [17] [18] [19] } \\
\text { meteorológicas [20] [21] [22] [23] }\end{array}$ \\
\hline \multirow{5}{*}{$\begin{array}{l}\text { Ingeniería y } \\
\text { Tecnología }\end{array}$} & $\begin{array}{l}\text { Sistema de estacionamiento inteligente } \\
{[24]}\end{array}$ \\
\cline { 2 - 2 } & Monitoreo de variables eléctricas [25] \\
\cline { 2 - 2 } & $\begin{array}{l}\text { Sistemas de localización inalámbrico } \\
\text { para robot móviles [26] }\end{array}$ \\
\cline { 2 - 2 } & $\begin{array}{l}\text { Sistema inalámbrico para medición de } \\
\text { iluminación [27] }\end{array}$ \\
\hline
\end{tabular}

Fuente: Autores.

A partir de la información suministrada en la tabla 2 , se decidió seleccionar como estudio de caso el sistema para monitoreo de variables meteorológicas en tierra, y para dicha selección se tuvo en cuenta que la Universidad Pedagógica y Tecnológica de Colombia cuenta desde el año 1962 con una estación meteorológica de tipo Climatológico Principal, en donde se miden variables como precipitación, evaporación, temperaturas en diferentes condiciones, humedad relativa, brillo solar, velocidad del viento, radiación global y presión.

Actualmente, en esta estación meteorológica la recopilación de los datos se hace de forma manual, donde un operador toma las medidas de los instrumentos cada 12 horas y los reporta al Instituto de Hidrología, Meteorología y Estudios Ambientales (IDEAM). Los datos recopilados desde la UPTC contribuyen a la consolidación de información sobre el estado y las dinámicas del medio ambiente en la ciudad de Tunja y en general del área operativa número 6, que corresponde a los departamentos de Boyacá y Casanare.

Dentro de la disponibilidad de los elementos de laboratorio de la UPTC, se encuentran los sensores de temperatura y humedad relativa, por tal motivo se decidió seleccionar estas variables para la implementación del estudio de caso. Esto con el propósito de capturar las variables desde la ETS UPTC 
elevando así el porcentaje de uso de la infraestructura y de igual forma cualificar la operación de la estación meteorológica UPTC automatizando sus procesos.

En tal sentido, este estudio promueve la interacción y el fortalecimiento de las redes de conocimiento interdisciplinarias dentro de la Universidad, a partir de la posibilidad de ofrecer una solución tecnológica con beneficio directo hacia el aspecto ambiental.

\section{B. Etapa Dos}

En esta etapa se esquematizó el sistema general para la captura de los datos de las variables meteorológicas, tal como se puede apreciar en la Fig. 1, donde se muestran dos bloques: uno para representar los dispositivos del nodo coordinador (ETS) y otro para los componentes del nodo sensor (Estación Meteorológica). Para tal efecto, se utilizó un enlace de comunicación inalámbrico punto a punto para el envío de los datos que serán procesados, almacenados y visualizados en el computador principal de la ETS-UPTC.
El nodo coordinador de la estación terrena satelital está ubicado en las coordenadas $5^{\circ} 33^{\prime} 19^{\prime \prime}$ Norte y $73^{\circ} 21^{\prime} 18^{\prime \prime}$ Este, en una altitud de 2735 m s.n.m. El nodo sensor está ubicado en $5^{\circ} 33^{\prime} 13^{\prime \prime}$ Norte y $73^{\circ} 21^{\prime} 19$ " Este, en una altitud de 2690 m s.n.m. La separación entre estos dos nodos es de aproximadamente 197 metros sin línea de vista.

\section{Etapa Tres}

En este apartado, se describe cada una de las unidades básicas del Nodo Sensor, el proceso de funcionamiento y el programa implementado para el funcionamiento del nodo sensor. En la Fig. 2, se ilustra el prototipo del esquema.

Unidad de sensado (Sensores): Permite la captura de información de las magnitudes físicas seleccionadas. Para el estudio de caso, se usó el sensor de referencia DHT11, el cual posee dos sensores resistivos (temperatura y humedad), se caracteriza por tener una señal digital calibrada y bajo consumo de potencia, el protocolo de comunicación maneja un único hilo (1-wire). En la Tabla 3, se muestran especificaciones de este sensor.

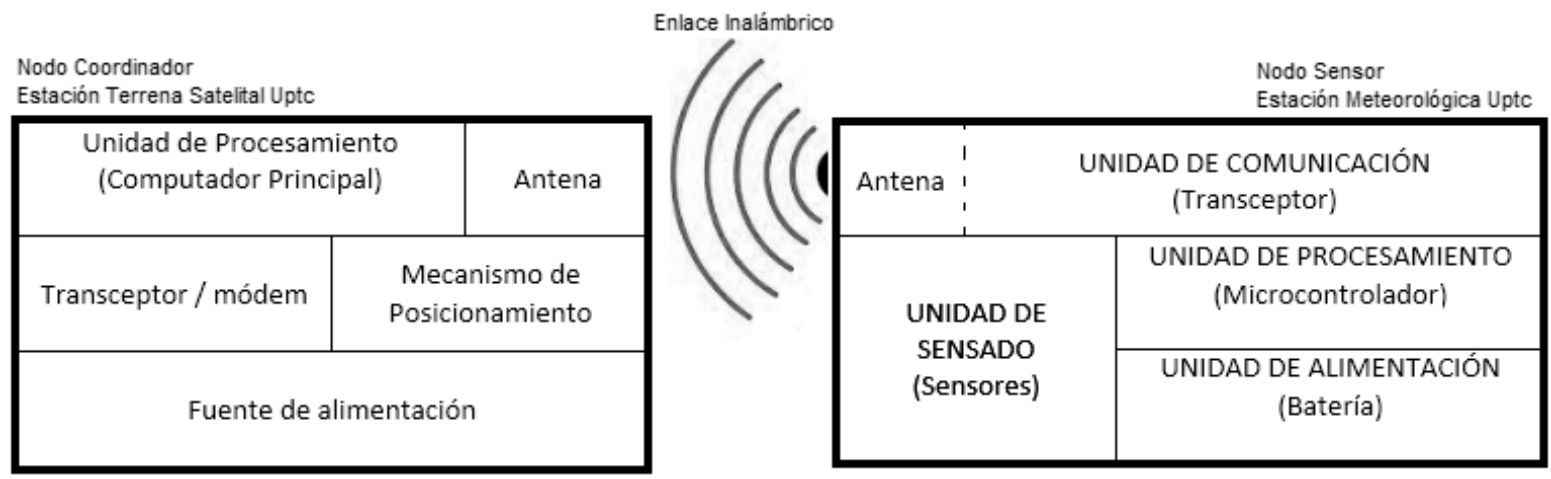

Fig. 1. Esquema General del Sistema de Captura de Variables Meteorológicas.

Fuente: Autores.

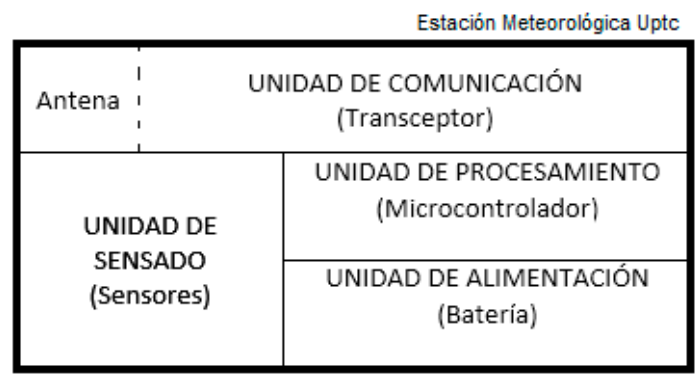

Fig. 2. Estructura del nodo sensor.

Fuente: Adaptado de "Componentes de nodos RIS" [16]. 
Tabla 3. Especificaciones sensor DHT11

\begin{tabular}{|l|l|c|c|}
\hline \multicolumn{1}{|c|}{ Parámetros } & Temperatura & \multicolumn{2}{|c|}{ Humedad relativa } \\
\hline Precisión & $\pm 2^{\circ} \mathrm{C}$ & \multicolumn{2}{|c|}{ De $0-50{ }^{\circ} \mathrm{C} \pm 5 \%$ de $\mathrm{HR}$} \\
\hline \multirow{2}{*}{$\begin{array}{l}\text { Rango de } \\
\text { medida }\end{array}$} & $0-50{ }^{\circ} \mathrm{C}$ & $20^{\circ} \mathrm{C}$ & $30-90 \% \mathrm{HR}$ \\
\cline { 2 - 4 } & & $25^{\circ} \mathrm{C}$ & $20-90 \% \mathrm{HR}$ \\
\hline Alimentación & $3.5-5.5 \mathrm{~V}$ \\
\hline $\begin{array}{l}\text { Corriente de } \\
\text { alimentación }\end{array}$ & \multicolumn{3}{|l}{$0.3 m \mathrm{HR}$ en medida, $60 \mu \mathrm{A}$ en espera } \\
\hline $\begin{array}{l}\text { Período de } \\
\text { muestreo }\end{array}$ & 2 segundos aproximadamente \\
\hline Resolución & \multicolumn{3}{|l|}{16 bits } \\
\hline
\end{tabular}

Unidad de procesamiento (Microcontrolador): Coordina todos los elementos que hacen parte del nodo sensor. Realiza la captura de las señales a través del sensor y usa el transceptor para enviarlas al nodo coordinador. Se empleó una placa Arduino Uno, que cuenta con un microcontrolador ATmega328P, 14 pines para entradas/salidas digitales, 6 entradas analógicas, un puerto In Chip Serial Programmer (ICSP), conexión para USB, conexión para alimentación eléctrica $(7$ - $12 \mathrm{~V})$. La programación de este módulo se realizó con el entorno de desarrollo integrado (Integreted Development Environment - IDE), basado en software libre, lo que representa gran facilidad para configuración y una ventaja para ser usado en proyectos académicos o de investigación [29].

Unidad de Comunicación (Transceptor): Permite el envío de los datos de forma inalámbrica hasta la ETS-UPTC. Teniendo en cuenta que la transmisión de los datos se hizo en banda $\mathrm{S}$, en los rangos de operación permitidos, con una topología de red punto a punto y se estimó que la distancia entre los puntos es de 197 metros, se utiliza un módulo XBee PRO S2B. Las especificaciones para este módulo [30] indican que en espacio abierto tiene un alcance de hasta 3200 metros, potencia de transmisión de hasta $17 \mathrm{dBm}$, frecuencia de operación de $2,4 \mathrm{GHz}$ para uso en aplicaciones Industriales Científicas y Médicas - ICM trae su propia antena integrada y requiere de $(2,7-3,6 \mathrm{~V})$ para alimentación. La configuración se realizó utilizando el programa XCTU, que se puede descargar de forma gratuita de la página web del fabricante [31].

Unidad de alimentación (Batería): Dados los requerimientos de alimentación del nodo sensor (7 - 12 $V$ ), la amplia disponibilidad y el bajo costo, se utilizó para el estudio de caso una batería alcalina de $9 \mathrm{~V}$.

Con las anteriores cuatro unidades y para el funcionamiento del nodo sensor, se implementó el código utilizando la Interfaz de Programación Arduino (Fig. 3). En este programa, se usó la librería DHT del sensor, la cual permite la comunicación entre la placa Arduino con los sensores de la familia DHT, el uso de la librería facilita la obtención de los valores de humedad en el aire y de la temperatura en el ambiente, permite leer los valores con variable tipo float. Con las instrucciones correspondientes, según la sintaxis de programación en Arduino, se determinan los pines de lectura de datos, la velocidad de lectura, las variables de almacenamiento, la validez de los valores leídos y la visualización de dichos valores a través del monitor serial del entorno de desarrollo de Arduino (IDE). Después de cada envío, se activa el modo de hibernación de pin para bajo consumo de energía.

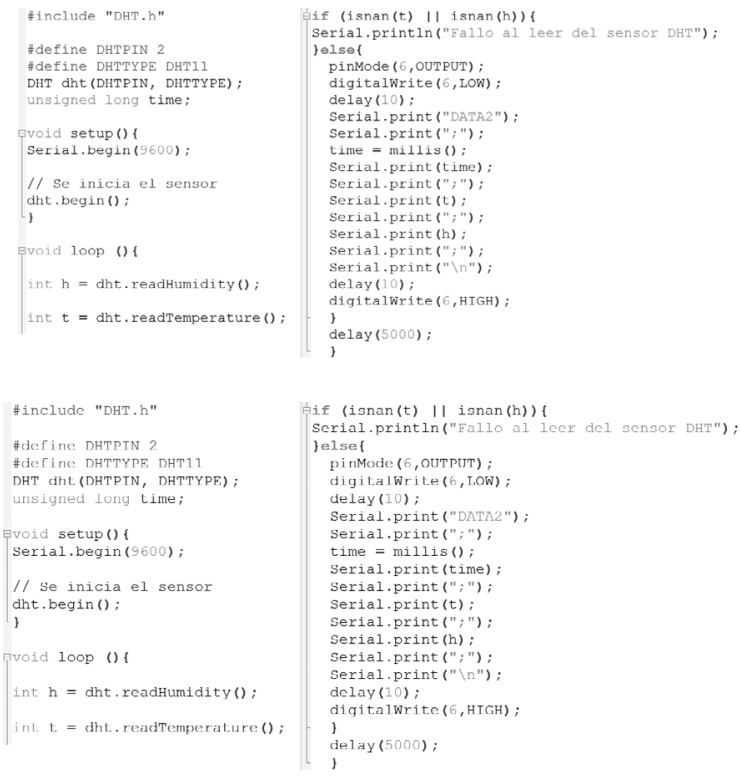

Fig. 3. Código para lectura de variables, implementado en el microcontrolador del Nodo Sensor. Fuente: Autores.

Finalmente, se presentan gráficamente las conexiones implementadas en el nodo sensor (Fig. 4).

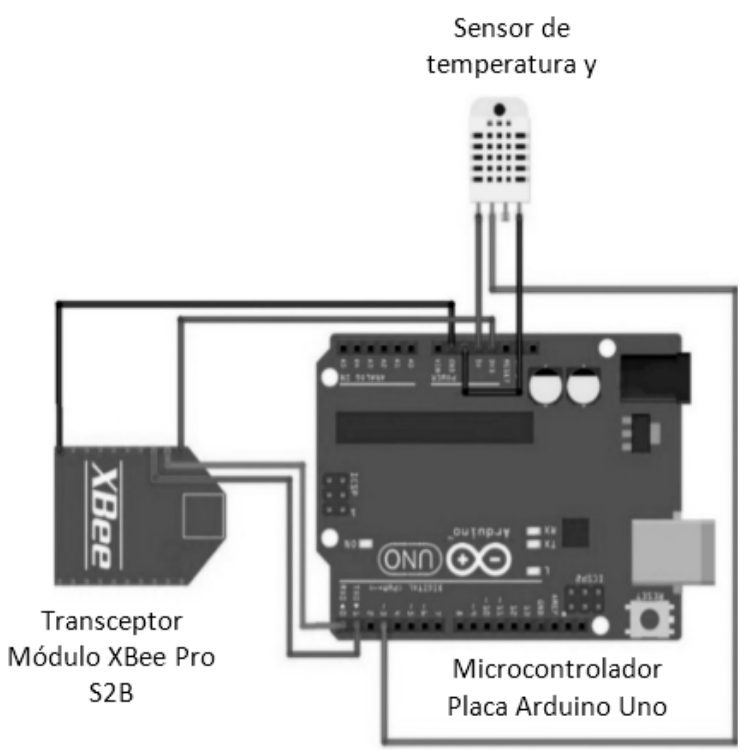

Fig. 4. Circuito Nodo Sensor. Fuente: Autores. 


\section{Etapa Cuatro}

Una vez realizada la captura de los datos de las variables seleccionadas (Temperatura ambiente y humedad relativa) se implementó la comunicación entre el nodo sensor y el nodo coordinador, para lo cual se realiza el test de comunicación entre el nodo sensor y el nodo coordinador. Cuyo escenario se describió en la etapa dos y muestra en la Fig. 5.

Para realizar el test de prueba se utilizó la opción Radio Range Test del software X-CTU y se usó como transceptor en la estación terrena un módulo XBee Pro S2B RPSMA conectado a la antena de banda S.
En este test se enviaron 100 paquetes de datos con separación de los nodos a distancias de: $5 \mathrm{~m}, 10 \mathrm{~m}$ y $50 \mathrm{~m}$ con línea de vista y a distancias de $5 \mathrm{~m}, 10 \mathrm{~m}$, $112 m$ y $196,52 m$ sin línea de vista; para cada una de las pruebas fue medido un indicador de intensidad de señal y la eficiencia de recepción de paquetes.

Para la última distancia señalada en el párrafo anterior, que corresponde a la distancia real del escenario propuesto, se obtuvo un RSSI de $-51 \mathrm{dBm}$ y una eficiencia en la recepción de paquetes del $96 \%$, garantizando una señal idónea y una estabilidad en la tasa de transferencia, tal como se evidencia en la Fig. 6.

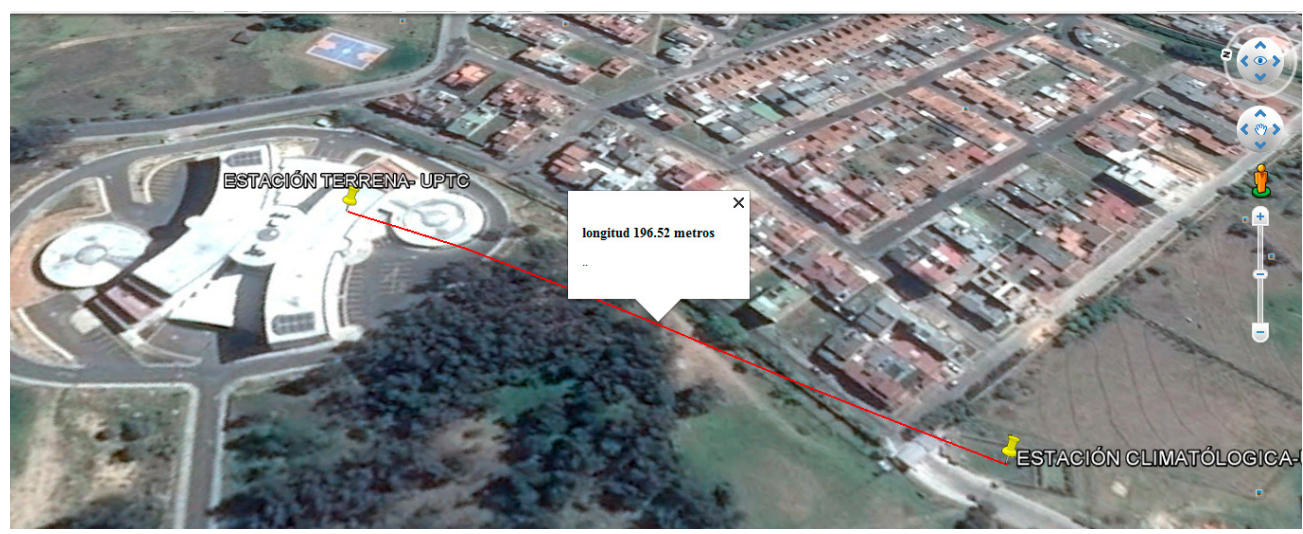

Fig. 5. Mapa con la ubicación geográfica y distancia entre las estaciones. Fuente: Adaptado a partir de (C) Google Earth.

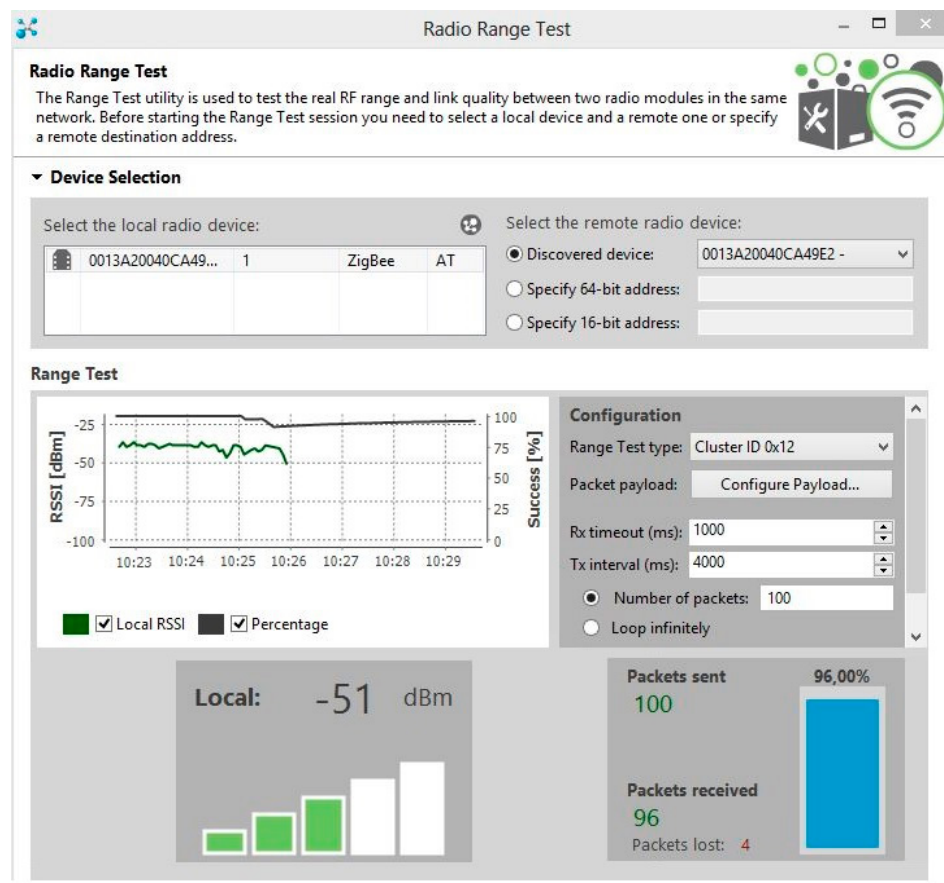

Fig. 6. Test de comunicación con el software X-CTU.

$$
\text { Fuente: Autores. }
$$




\section{E. Etapa Cinco}

En el nodo coordinador se implementó una interfaz gráfica para validar los datos enviados por el nodo sensor. Para la adquisición y visualización de dichos datos en el computador principal de la Estación Terrena, se utilizó inicialmente Processing, lenguaje compatible con Arduino. Los datos fueron leídos a través del puerto serial, mostrados en pantalla y guardados en un archivo de texto plano, para que puedan ser utilizados posteriormente.

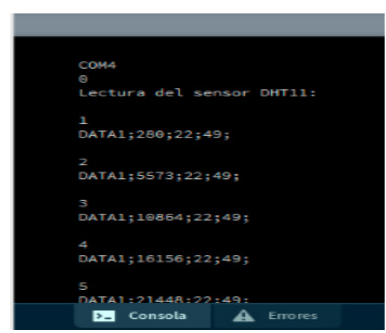

(a)

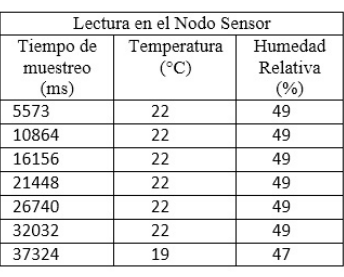

(b)
Fig. 7. (a) Processing: Entorno para recuperación y visualización de los datos provenientes del nodo sensor. (b) Relación de variables y datos.

$$
\text { Fuente: Autores. }
$$

En la Fig. 7, se observa una muestra de los datos recibidos, cuyo formato incluye encabezado, valor de tiempo en milisegundos, valor de temperatura $\left({ }^{\circ} \mathrm{C}\right)$ y valor de humedad relativa (\%).

Adicionalmente, se elaboró la interfaz gráfica de visualización de datos con las variables meteorológicas humedad relativa y temperatura ambiente (Fig. 8). Se gestionaron los datos para determinar el valor promedio de cada una de las variables y simular una alarma visual que responda a un umbral específico.

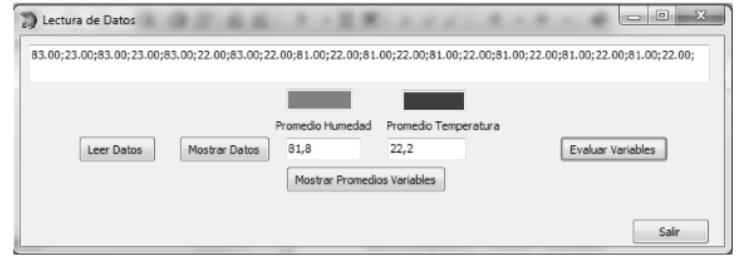

Fig. 8. Interfaz gráfica de visualización de datos. Fuente: Autores.

La ejecución de las fases anteriores, permitió la correspondiente implementación del sistema completo, tal como se ilustra en la Fig. 9.

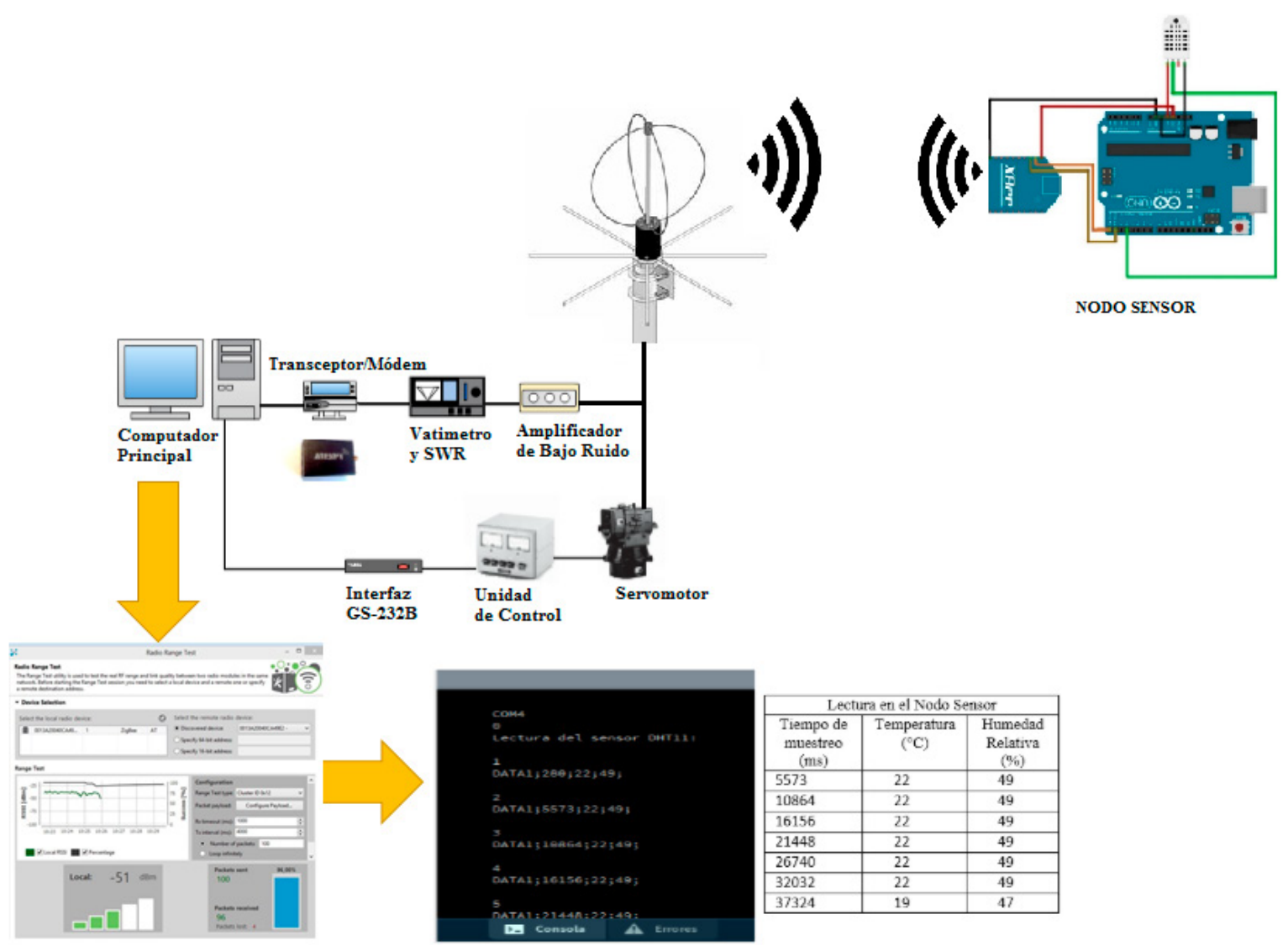

Fig. 9. Sistema Implementado. Fuente: Autores. 


\section{III.RESULTADOS}

La metodología utilizada en fases para la construcción del sistema general permitió estructurar la ejecución del proyecto para obtener los resultados que presentados en este artículo.

En el proceso investigativo se logró la implementación de un nodo sensor que integrado con la ETSUPTC, permitió el monitoreo de variables meteorológicas manteniendo en ejecución las funciones de seguimiento a satélites de órbita baja.

También, se logró el diseño de los nodos (sensor y coordinador) con la metodología de diagrama de bloques, que permitió evidenciar el correcto funcionamiento e integración de los diversos dispositivos para la captura de las variables meteorológicas.

Además, se implementó el código para capturar los datos de las variables meteorológicas, utilizando el sensor (DHT11) y el microcontrolador ATmega $328 P$ (Arduino) y de igual forma el código para la interfaz gráfica de los datos recibidos en el nodo coordinador.

De otro lado, se evidenció el incremento en el porcentaje de uso de la ETS-UPTC, tomando el registro de tiempo de operación con sólo funciones de seguimiento a satélites durante un período de 24 horas. Estos datos se compararon con operaciones tanto satelitales como de monitoreo de señales en tierra, durante el mismo período de tiempo (Fig. 10).

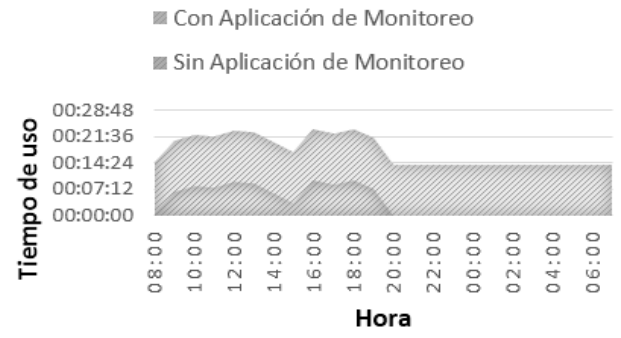

Fig. 10. Comparativo de tiempo de uso de la estación terrena por día. Fuente: Autores.

La aplicación del Sistema de Monitoreo de señales en tierra representó un incremento estimado del $20 \%$, en sus niveles de uso diario para el caso de la ETSUPTC (Fig. 11). Cabe resaltar que, se puede seguir incrementando la frecuencia de envío de las mediciones y el número de variables a monitorear, y por lo tanto, este porcentaje puede ser mayor.
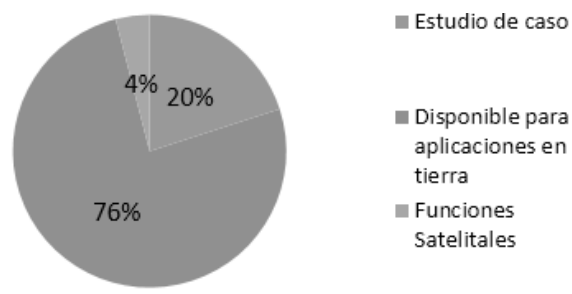

Fig. 11. Porcentaje diario de uso de la estación terrena de la UPTC. Fuente: Autores.
El factor diferenciador del proyecto desarrollado en relación a otros sistemas de monitoreo, radica en el uso de estaciones terrenas satelitales que realicen funciones distintas a las relacionadas con seguimiento a satélites y a la posibilidad de dar un nuevo enfoque respecto al uso de la infraestructura en la ETS-UPTC.

\section{Conclusiones}

El desarrollo de esta investigación, constituye un aporte significativo en la generación de alternativas desde las ciencias naturales, para incrementar el porcentaje de uso de la infraestructura de la ETSUPTC. De igual modo se puede evidenciar que se logró describir las fases desarrolladas para implementar un sistema de monitoreo de señales en tierra.

Con los datos arrojados en la primera fase (Tabla 1), se puede afirmar que existen actualmente varias alternativas tanto en ciencias agrícolas, ciencias de la salud y ciencias de la ingeniería y tecnología para ejecutar el monitoreo de variables que permitan la optimización y el aprovechamiento de la ETS-UPTC.

Dentro de los aspectos visiónales de la Universidad Pedagógica y Tecnológica de Colombia, se contempla el desarrollo social sustentable, la responsabilidad social y pertinencia, tanto a nivel regional (Boyacá y Casanare), como a nivel nacional. En tal sentido, el proyecto del cual se ha derivado el presente estudio, tiene un aporte potencial en los procesos de consolidación y distribución de la información sobre el comportamiento de variables meteorológicas, con el monitoreo y reporte sistematizado de datos, que podrían ser utilizados como apoyo al sistema de prevención de riesgos.

Finalmente, cabe resaltar que cuando se realiza un monitoreo de las variables en horas fijas del día y se almacenan sistemáticamente los valores correspondientes en un centro recolector de datos, estas observaciones podrían utilizarse para un amplio espectro de análisis meteorológicos con utilidad inmediata, y especialmente para la elaboración de mapas meteorológicos, que permitan hacer diagnósticos y formular los pronósticos del tiempo con mayor cobertura y precisión.

\section{Agradecimientos}

Al finalizar este estudio se hace extensivo los agradecimientos al grupo de Investigación en Informática, Electrónica y Comunicaciones (INFELCOM) y a la Vicerrectoría de Investigaciones y Extensión de la Universidad Pedagógica y Tecnológica de Colombia por el apoyo y la financiación que permitieron la realización de este proyecto que se convierte en un aporte a la comunidad académica en tecnologías de las comunicaciones satelitales, gracias al uso de la ETS-UPTC, con fines de investigación, desarrollo, innovación y apropiación social del conocimiento. 


\section{REFERENCIAS}

[1] R. Neri, Comunicaciones por Satélite. México D.F., Mex: Thomson, 2003.

[2] O. F. Vera, "Análisis de desempeño del protocolo ax.25 en el enlace de la estación terrena satelital UPTC", Univ. Pedagógica y Tecnológica de Colombia, Tunja, 2015.

[3] L. Drewes, El sector espacial argentino: Instituciones referentes, proveedores y desafíos. 1er. ed. Benavídez: ARSAT - Empresa Argentina de Soluciones Satelitales, 2014.

[4] Instituto Nacional de Pesquisas Espacias - INPE. "Programa de Desenvolvimiento de Cubesat-NanosatC-Br1”. INPE/NanosatCBR. http://www.inpe.br/crs/nanosat/index.php [acceso: 16 de marzo de 2016]

[5] J. D. Puerto, "Universidad Sergio Arboleda y GENSO, en la Órbita de la Innovación". Ingeniería e Industria Aeroespacial. http:// ingenieriaindustrialsergioarboleda.blogspot.com/2012/05/universidad-sergio-arboleda-y-genso-en.html [acceso: marzo 16 de 2016]

[6] L. E. Aparicio y C. Cerquera, "Diseño, simulación e implementación de una estación terrena CUBESAT UD - USCO", Convenio de cooperación académica Universidad Surcolombiana-Universidad Distrital Francisco José de Caldas, Neiva, 2014

[7] O. Pérez y M. Á. Hernández, "Diseño e Implementación de una Estación Terrena en la Unipanamericana Bogotá para seguimiento de Satélites en la banda de Radioaficionados", Tesis grado, Dept. Ing. Telcom., Unipanamericana., Bogotá, D.C., Col, 2014.

[8] J. E. Espíndola y J. A. Mesa, "Implementación de estación terrena para seguimiento a picosatélites de órbita LEO", Ventana Informática, vol. 26, no. 1, pp. 77-91, Ene. 2012.

[9] J. E. Espíndola, C. A. Nonsoque y D. Molano, "Montaje e implementación de una estación terrena para el seguimiento de satélites de órbita baja” en Tenth Latin American and Caribbean Conference for Engineering and Technology - Universidad Tecnológica de Panamá, Panamá City, Jul 24-27, 2012.

[10] O. Vera y J. Mesa, "Modelo para el sistema de posicionamiento de las antenas de la estación terrena satelital de la Universidad Pedagógica y Tecnológica de Colombia", Ingenio Magno, vol. 6, $\mathrm{n}^{\circ}$ 1, pp. 24-33, Ene. 2015.

[11] J. E. Espíndola, R. Ferro y J. A. Mesa, "Direccionamiento automá tico de antenas en estaciones terrenas de seguimiento a picosatelites", Tecnura, vol. 17, n 35, p. 26-37, Ene. 2013.

[12] R. Hernández, C. Fernández y P. Baptista, Metodología de la In vestigación, México D.F., Mex: McGraw-Hill, 2014

[13] Cuadro Nacional de Atribución de Bandas de Frecuencia Actualización julio 2016, Agencia Nacional del Espectro, Colombia, 2016

[14] S. Sendra, F. Llario, L. Parra y J. Lloret, "Smart Wireless Sensor Network to Detect and Protect Sheep and Goats to Wolf Attacks", Recent Advances in Communications and Networking Technology, vol. 2, no. 2, pp. 91-101, Dec. 2013. https://doi.org/10.2174/221174 07112016660012

[15] C. Lozoya, A. Aguilar, and C. Mendoza, "Service Oriented Design Approach for a Precision Agriculture Datalogger", IEEE Latin America Transactions, vol. 14, no. 4, pp. 1683-1688, Apr. 2016 https://doi.org/10.1109/TLA.2016.7483501

[16] M. Manzano, S. Montesinos, J. A. Carazo y C. Vázquez, "Las redes inalámbricas de sensores y su aplicación en el sector agroalimentario", Revista de la Alta Tecnología y la Sociedad, vol. 7, no. 1, pp. 1-7, Oct. 2015. Disponible en https://drive.google.com/drive/folder s/0B4GS5FQQLif9QURhaVU4aWVJdDQ

[17] A. Pilco, V. Zavala, O. Martínez and T. Flores, "Implementation of the WSN prototype to monitoring of patients on ESPOCH comprehensive health center", in IEEE Thirty Fifth Central American and Panama Convention - CONCAPAN XXXV, Tegucigalpa, Honduras, Nov. 11-13, 2015. https://ieeexplore.ieee.org/ document/7428479

[18] M. Vázquez, J. Garibaldi, J. Nieto, and J. de Dios, "Model for Personalization of Mobile Health Systems for Monitoring Patients with Chronic Disease", IEEE Latin America Transactions, vol. 14, no. 2, pp. 965-970, Mar. 2016. https://doi.org/10.1109/ TLA. 2016.7437247

[19] J. P. Tello, O. Manjarrés, M. Quijano, A. Blanco, F. Varona, and M. Manrique, "Remote Monitoring System of ECG and Human Body Temperature Signals", IEEE Latin America Transactions, vol. 11, no. 1, pp. 314-318, Apr. 2013. https://doi.org/10.1109/ TLA.2013.6502822
[20] H. Kaschel y J. Pérez, "Monitoreo ubicuo de salud en tiempo real con WBSN", Revista Chilena de Ingeniería, vol. 22, no. 2, pp. 169-176, Apr. 2014. http://dx.doi.org/10.4067/S071833052014000200003

[21] J. R. Ruiz, C. E. Vargas, E. Villarreal, E. Torres, D. Díaz, y F. Univio, "Sistema para monitoreo inalámbrico de niveles de gas metano, temperatura y monóxido de carbono en túneles viales", Revista Entérese Boletín Científico Universitario, vol. 32, no. 1, pp. 38-43, Jun. 2012.

[22] G. Espitia y Á. Mejía, "Plataformas tecnológicas aplicadas al monitoreo climático", Prospectiva, vol. 11, no. 2, pp. 78-87, Jul. 2013.

[23] J. A. Blanco y J. N. Pérez, "Redes inalámbricas de geosensores aplicadas en sistemas de observación y monitoreo ambiental”, Gerencia Tecnológica Informática, vol. 11, no. 29, pp. 59-68, Ene. 2012.

[24] M. Quiñones, V. González, L. Quiñones, C. Valdivieso, and W. Yaguana, "Design of a smart parking system using wireless sensor network", In: 10th Iberian Conference on Information Systems and Technologies (CISTI), Aveiro, portugal, Jun. 17-20, 2015. https://doi.org/10.1109/CISTI.2015.7170430

[25] F. J. Enríquez, E. Sifuentes, J. Cota, L. H. Rascón, J. F. Estrada, y F. J. López, "Sistema de monitoreo de variables eléctricas V, I y P", CULTCyT, vol. 12, no. 57, Especial 1, pp. 28-36, Sept. 2015. Disponible en http://erevistas.uacj.mx/ojs/ index.php/culcyt/article/viewFile/769/736

[26] A. Canedo-Rodríguez, J. Rodríguez, V. Álvarez-Santos, R. Iglesias y C. Regueiro, "Mobile Robot Positioning with 433 $\mathrm{MHz}$ Wireless Motes with Varying Transmission Powers and a Particle Filter", Sensors, vol. 15, no. 5, pp. 10194-10220, Apr. 2015. https://doi.org/10.3390/s150510194

[27] E. Sifuentes, R. González, G. Bravo, y R. G. Moreno, "Nodo sensor inalámbrico para medir iluminación”, CULTCyT, vol. 12 , no. 56, May. 2015.

[28] DHT11 Humidity \& Temperature Sensor, Osepp Electronics. [En línea]. Disponible en https://www.mouser.com/ds/2/758/ DHT11-Technical-Data-Sheet-Translated-Version-1143054. pdf [acceso: 04 de Febrero de 2017].

[29] Arduino, "Arduino Uno \& Genuino Products". Store Arduino. https://www.arduino.cc/en/main/arduinoBoardUno [acceso: 04 de Febrero de 2017].

[30] ZigBee RF Modules XBEE2, XBEEPRO2, PROs2B. User Guide, Digi International, [En línea]. Disponible en https://www. digi.com/resources/documentation/digidocs/pdfs/90000976. pdf [acceso: 04 de Febrero de 2017].

[31] Digi International Inc. (Abril, 2017) XCTU-OSX, [En línea]. Disponible en https://www.digi.com/resources/documentation/digidocs/90001526/tasks/t_download_and_install_ xctu.htm

Jairo Alonso Mesa Lara es Ingeniero Electrónico, Magíster en Ciencias de la Información y las Comunicaciones, Docente de planta, Universidad Pedagógica y Tecnológica de Colombia, Facultad de Ingeniería, Escuela de Ingeniería de Sistemas y Computación, Tunja, Boyacá, Colombia. https://orcid.org/0000-00017303-3055

Jenny Amparo Rosales Agredo es Ingeniera de Sistemas, Magíster en Ingeniería Industrial, Docente de planta, Universidad Pedagógica y Tecnológica de Colombia, Facultad de Ingeniería, Escuela de Ingeniería Electrónica, Tunja, Boyacá, Colombia. https:// orcid.org/0000-0001-8004-4369

María Paula Maury Atencia es Ingeniera Electrónica, Magister en Tecnología Informática, Docente ocasional, Universidad Pedagógica y Tecnológica de Colombia, Facultad de Estudios a Distancia, Escuela de Ciencias Tecnológicas, Tunja, Boyacá, Colombia. https://orcid.org/0000-0002-5873-8759 